\title{
A Multi-Scale Compressed Sensing Algorithm Based on Variational Mode
}

\author{
Shuyao Tian ${ }^{1}$, Pai Zhang ${ }^{2}$, Hongju Lin ${ }^{3}$ \\ ${ }^{1}$ College of Electronics and Control Engineering, North China Institute of Aerospace Engineering, Langfang, \\ China \\ ${ }^{2}$ College of Intelligence and Information Engineering, Tangshan University, Tangshan, China
}

Received: March 17, 2020. Revised: July 17, 2020. 2nd Revised: August 26, 2020.

Accepted: September 18, 2020. Published: September 22, 2020.

\begin{abstract}
The compressed sensing algorithm based on the hybrid sparse base (TFWBST+wave atom) usually uses two kinds of image sparse transformations to realize the sparse representation of structure and texture respectively. However, due to the lack of constraints on image texture and structure and the lack of orthogonality of the two sparse bases, the sparse coefficient of structure and the sparse coefficient of texture after transformation are often not good enough to reflect their respective components, that is, the texture coefficient often loses the detail information of texture. To overcome this phenomenon, this paper combines the compressed sensing algorithm based on hybrid base with the layered variational image decomposition method to form the variational multi-scale compressed sensing, which is to establish the CS image reconstruction model with minimal energy functional. The layered variational image decomposition decomposes image into different feature components by minimizing energy functional. The reconstruction of each layer by compressed sensing algorithm is very suitable for texture and detail reconstruction. In this model, TFWBST transform and wave atom are combined as a joint sparse dictionary, and the image decomposition is carried out under the $(B V, G, E)$ variational framework, which is introduced into multi-scale compressed sensing technology to reconstruct the original image. In this new functional, TFWBST transform and wave atom are used to represent structure and texture respectively, and multiscale $(B V, G, E)$ decomposition which can decompose an image into a sequence of image structure,
\end{abstract}

texture and noise is added for restricting image parts. Experiments show that the new model is very robust for noise, and that can keep edges and textures stably than other multi-scale restoration and reconstruction of images.

Keywords-Variational model, Multi-scale, Compressed sensing, CS reconstruction.

\section{INTRODUCTION}

C ompressed Sensing is a new sampling theorem, it points

out that if a signal can be compressed under some conditions, that a very accurate reconstruction can be obtained from a relatively small number of nontraditional samples[1].

Since exploiting a prior knowledge of the original signals is critical to the success of CS theory, numerous studies have been performed to build more realistic models for real-world signals[2]. Conventional CS recovery exploits the 11-norm based sparsity of a signal and the resulting convex optimization problems can be efficiently solved by the class of surrogatefunction based methods. More recently, the concept of sparsity has evolved into various sophisticated forms. The wavelet transform has been proven to be powerful in many signal and image processing applications such as compression, denoise, image enhancement, and image feature extraction.wavelets are not optimal in capturing the two-dimensional singularities 
found in images. Therefore, several transforms have been proposed for image signals that have incorporated directionality and multiresolution and hence, could more efficiently capture edges in natural images[3].

On the basis of compressed sensing,the paper presents multiscale compressed sensing. We proposes a new image tranform based on wavelets and shear filter,referred as tight frame wavelet based Shearlet transform (TFWBST). we first used wavelet transform to perform multiscale decomposition of the image. Then in the high frequency subband, we used shear filter to perform multi-direction decomposition of the image, which can represent the image more sparsely. TFWBST transform and wave atom are combined as a joint sparse dictionary, and the image decomposition is carried out under the $(B V, G, E)$ variational framework, which is introduced into multiscale compressed sensing technology to reconstruct the original image. The numerical experiments demonstrate that multiscale compressed sensing can give better quality reconstruction than a literal deployment of the compressed sensing methodology.

\section{TFWBST SPARSE REPRESENTATION}

\section{A The Construction Principle of TFWBST}

The contour, edge and some texture of the natural image are the discontinuities of the image, which are often manifested as point singularity, curve singularity or plane singularity. According to the research results of physiologists on human visual system and the statistical model of natural image, a "sparsest" image representation method should have three basic characteristics: multi-resolution, locality and directionality. In spatial domain and frequency domain, the primary function of sparse representation method has localized performance. TFWBST is constructed based on the above ideas. The realization is divided into two stages: the multi-scale decomposition of the two-dimensional orthogonal wavelet basis and the directional subdivision of the high-frequency coefficients by the shear operation of the shear filter. A twodimensional separable orthogonal wavelet basis spanned by an one-dimensional wavelet has a square support interval. Under different resolution, its support interval is a square of different sizes. In fact, the process of two-dimensional wavelet approaching the singular curve of the image is essentially the process of using points to approach the line[4]. However, when the decomposition scale gets smaller, the number of non-zero wavelet coefficients increases exponentially, and a large number of non-negligible coefficients appear, which finally can not be sparse to represent the original image.

To make full use of the geometric regularity of the image[5], the square support interval of the two-dimensional orthogonal wavelet basis is further divided in the direction by referring to the mode of direction decomposition of the discrete shear filter in the frequency domain, and the new "basis" is expected to have a structure of direction and length and width transformation. The discrete shear filter is a localized window function on symmetric trapezoid pairs, which is constructed on the basis of continuous shear waves. Through the study of synthetic wavelet theory, the affine system can be used to combine geometry and multi-scale analysis to construct shear waves. When the dimension $n=2$, the definition of continuous shear wave transform is as follows:

$$
S H_{\psi} f(a, s, t)=\left\langle f, \psi_{a, s, t}\right\rangle
$$

where $a>0, s \in R, t \in R^{2}, \psi_{a, s, t}$ is called shear wave, which is expressed as

$$
\psi_{a, s, t}(x)=\left|\operatorname{det} M_{a, s}\right|^{-\frac{1}{2}} \psi\left(M_{a, s}^{-1} x-t\right)
$$

where $M_{a, s}=\left(\begin{array}{cc}a & \sqrt{a s} \\ 0 & \sqrt{a}\end{array}\right), M_{a, s}=B_{s} A_{a}, A_{a}=\left(\begin{array}{cc}a & 0 \\ 0 & \sqrt{a}\end{array}\right)$, which is an anisotropic expansion matrix. $B_{S}=\left(\begin{array}{ll}1 & S \\ 0 & 1\end{array}\right)$ is the shear matrix.

Here, the generation function $\psi$ is localized and meets the allowable conditions. For the $f \in R^{2}$, there is

$$
f=\int_{R^{2}} \int_{-\infty}^{\infty} \int_{0}^{\infty}\left\langle f, \psi_{a, s, t}\right\rangle \psi_{a, s, t} \frac{d a}{a^{3}} d s d t
$$

Specifically, $\xi=\left(\xi_{1}, \xi_{2}\right) \in R^{2}, \xi_{2} \neq 0, \hat{\psi}$ is defined as

$$
\hat{\psi}(\xi)=\hat{\psi}_{1}\left(\xi_{1}\right) \hat{\psi}_{2}\left(\frac{\xi_{2}}{\xi_{1}}\right)
$$

where $\hat{\psi}_{1}$ and $\hat{\psi}_{2}$ are smooth functions, and their support intervals are sup $p \hat{\psi}_{2} \in[-1,1]$.

In addition, $\sup p \hat{\psi}_{1} \in[-1 / 2,1 / 16] \cup[1 / 16,1 / 2]$. Assuming $\sum_{j \geq 0}\left|\hat{\psi}_{1}\left(2^{-2 j} \omega\right)\right|^{2}=1$, under the above conditions, where $j>0,-2^{j} \leq l \leq 2^{j}-1, k \in Z^{2} \quad, \quad$ a wedge piece on $D_{0}=\left\{\left(\xi_{1}, \xi_{2}\right) \in R^{2},\left|\xi_{1}\right| \geq 1 / 8,\left|\xi_{2} / \xi_{1}\right| \leq 1\right\} \quad$ is $\quad$ formed. Similarly, the function $\psi_{j, l, k}^{1}$ can be constructed to form a wedge piece on $D_{1}=\left\{\left(\xi_{1}, \xi_{2}\right) \in R^{2},\left|\xi_{2}\right| \geq 1 / 8,\left|\xi_{1} / \xi_{2}\right| \leq 1\right\}$. 
In frequency domain, the $\hat{\psi}_{a, s, t}$ is defined as

$$
\hat{\psi}_{a, s, t}\left(\xi_{1}, \xi_{2}\right)=a^{\frac{3}{4}} e^{-2 \pi i \xi t} \hat{\psi}_{1}\left(a \xi_{1}\right) \hat{\psi}_{2}\left(a^{-\frac{1}{2}}\left(\frac{\xi_{2}}{\xi_{1}}-s\right)\right)
$$

Therefore, for each shear wave $\hat{\psi}_{a, s, t}$, the frequency domain support is as follows:

$\sup p \hat{\psi}_{a, s, t} \subset\left\{\left(\xi_{1}, \xi_{2}\right), \xi_{1} \in\left[-2^{2 j-1},-2^{2 j-4}\right] \cup\left[2^{2 j-4}, 2^{2 j-1}\right]|| 2^{-j}+\xi_{2} / \xi_{1} \mid \leq 2^{-j}\right\}$

That is, each element $\hat{\psi}_{a, s, t}$ is supported on a trapezoid pair, the approximate size is $2^{2 j} \times 2^{j}$, and the direction is along a straight line with a slope of $l 2^{-j}$. Based on the above analysis of shear wave support interval, a discrete shear filter is constructed [6]. It is still assumed that $\xi=\left(\xi_{1}, \xi_{2}\right) \in R^{2}$,

$$
\omega_{j, l}^{d}=\left\{\begin{array}{c}
\hat{\psi}_{2}\left(2^{j} \frac{\xi_{2}}{\xi_{1}}-l\right) x_{D_{0}}(\xi)+\hat{\psi}_{2}\left(2^{j} \frac{\xi_{1}}{\xi_{2}}-l+1\right) x_{D_{1}}(\xi), l=-2^{j} \\
\hat{\psi}_{2}\left(2^{j} \frac{\xi_{2}}{\xi_{1}}-l\right), 1-2^{j} \leq l \leq 2^{j}-2 \\
\hat{\psi}_{2}\left(2^{j} \frac{\xi_{2}}{\xi_{1}}-l\right) x_{D_{0}}(\xi)+\hat{\psi}_{2}\left(2^{j} \frac{\xi_{1}}{\xi_{2}}-l-1\right) x_{D_{1}}(\xi), l=2^{j}-1
\end{array}\right.
$$

where $\hat{\psi}_{2}, D_{0}, D_{1}$ has been defined. For $1-2^{j} \leq l \leq 2^{j}-2$, each $\omega_{j, l}^{d}(\xi)$ is a window function localized on a trapezoid pair, as shown in Fig.1.
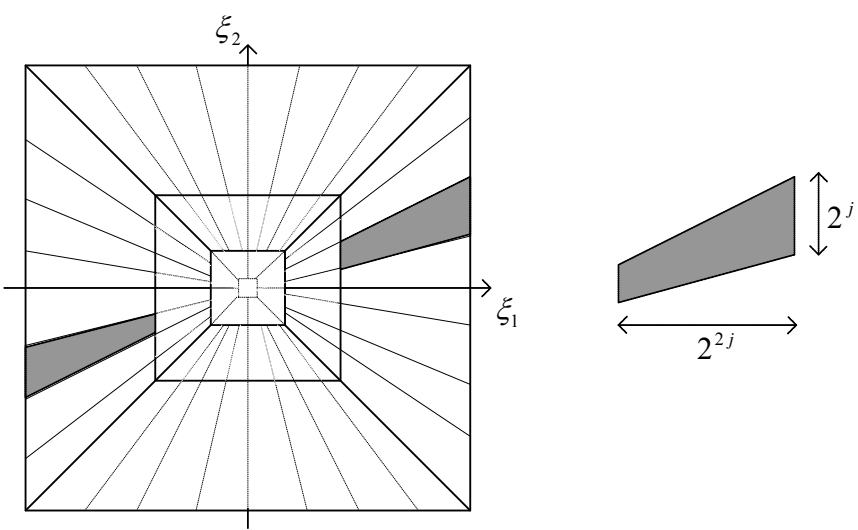

Fig. 1. The frequency domain support of Shearlet window function

When $l=-2^{j}$ or $l=2^{j}-1$, at the junction of $D_{0}$ and $D_{1}$, $\omega_{j, l}^{d}(\xi)$ is the superposition of these two functions, and $\omega_{j, l}^{d}(\xi)$ is called discrete shear filter. On the pseudo polar lattice [7], the further processed $\omega_{j, l}^{d}(\xi)$ can divide the high frequency coefficients into components in different directions.

\section{B Wave Atoms}

Texture, as the background component of the image, is a special structure of image information, which can not be represented sparsely in the wavelet domain or curvelet [8] domain. In texture image processing, the wave atom is widely used and has achieved good results.

The wave atom is a kind of variant wavelet packet, which can also be regarded as the "interpolation" between wavelet and Garbo. Its support domain satisfies the parabolic proportion relationship: wavelength $\approx(\text { diameter })^{2}$. A formal definition of a two-dimensional wave atom will be given. The wave atom is recorded as $\varphi_{\mu}(x)$, and its subscript $\mu=(j, m, n)=\left(j, m_{1}, m_{2}, n_{1}, n_{2}\right) \quad, \quad j, m_{i}, n_{i} \in Z(i=1,2)$. $\left(x_{\mu}, \xi_{\mu}\right)$ is regarded as any point in the phase space and satisfies the relation

$$
x_{\mu}=2^{-j} n, \xi_{\mu}=\pi 2^{j} m, C_{1} 2^{j} \leq \max \left|m_{i}\right| \leq C_{2} 2^{j}
$$

Among them, $C_{1}, C_{2}>0$, which are constants. $x_{\mu}, \xi_{\mu}$ represent the center of wave atom in space domain and frequency domain respectively. The localization condition of the wave atom near the $\left(x_{\mu}, \xi_{\mu}\right)$ point in the phase space must also be satisfied [9].

\section{MULTI-SCALE COMPRESSED SENSING ALGORITHM BASED ON LAYERED VARIATIONAL IMAGE DECOMPOSITION}

\section{A Multi-Scale Image Decomposition Algorithm Based on Layering $(B V, G, E)$}

A new single parameter $(B V, G, E)$ ternary decomposition model is proposed, and then the single parameter in the model is selected as a changing binary sequence to get the multi-scale decomposition of the image, and the convergence of the multiscale decomposition is proved. Note that the ternary decomposition model proposed by Aujol and Chambolle is not directly selected in this paper, because there are three parameters in this model that need to be adjusted. A single parameter in the new model proposed in this paper is enough to control the scale of the extracted structure, texture and noise.

The ternary variational decomposition model of the new single parameter $(B V, G, E)$ is defined as follows :

$\inf _{u \in B V, v \in G, \omega \in E}\left\{|\mathrm{u}|_{B V}+\lambda\|f-u-v-\omega\|_{L^{2}}^{2}+\|v\|_{G}+\|\omega\|_{E}\right\}$

where $\lambda>0$ is the adjustment parameter. By solving the minimization problem (9), the image $f$ is decomposed into four parts $f=u+v+\omega+r$, where $u \in B V$ represents the structural part of the image, $v \in G$ represents the texture part of the image, $\omega \in E$ represents the noise of the image, and $r=f-u-v-\omega$ represents the residual after decomposition. 
Model solving: CS reconstruction is carried out by using the introduced layered variational decomposition algorithm. In this paper, the alternating iterative algorithm is used to solve the minimization problem (9).

$v$ and $\omega$ are fixed, and $u$ is solved by the following minimization problem

$$
\inf _{u \in B V}\left\{\left.u\right|_{B V}+\lambda_{0} 2^{k+1}\left\|r_{k}-u-v-\omega\right\|_{L^{2}}^{2}\right\}
$$

$u$ and $\omega$ are fixed, and $v$ is solved by the following minimization problem

$$
\inf _{\omega \in E}\left\{\lambda_{0} 2^{K+1}\left\|r_{K}-u-v-\omega\right\|_{L^{2}}^{2}+\|\omega\|_{E}\right\}
$$

$u$ and $v$ are fixed, and $\omega$ is solved by the following minimization problem:

$$
\inf _{\mathrm{v} \in G}\left\{\lambda_{0} 2^{K+1}\left\|r_{K}-u-v-\omega\right\|_{L^{2}}^{2}+\|v\|_{G}\right\}
$$

The coupling problems (10) - (12) are solved respectively.

First, the minimization problem (10) is solved. Using the projection algorithm[10] proposed by Chambolle in the dual frame, the solution of the minimization problem (10) can be written as follows:

$$
u_{k+1}=r_{k}-v-\omega-\operatorname{Pr} o_{G_{1 / 2} \lambda_{K}}\left(r_{k}-v-\omega\right)
$$

where $\operatorname{Pr} 0 j_{G_{I / 2} \lambda_{K}}\left(r_{k}-v-\omega\right)$ represents the projection of $\left(r_{k}-v-\omega\right)$ on a closed convex set $\left\{\theta:\|\theta\|_{\mathrm{G}} \leq 1 / 2 \lambda_{K}\right\}$. Under the discrete condition, calculating $\operatorname{Pr} o j_{\left.G_{1 / 2} \lambda_{K}\right)}\left(r_{k}-v-\omega\right)$ is equivalent to solving the following minimization problem

$$
\min _{\xi \in C_{\mathrm{c}}^{1}\left(\Omega, R^{2}\right)}\left\{\left|\operatorname{div}(\xi)-2 \lambda_{k}\left(r_{k}-v-\omega\right) \|_{L^{2}}^{2},\right| \xi_{i, j} \mid \leq 1, i=1, \ldots N\right\}
$$

The Euler Lagrange equation of functional (14) is as follows:

$$
-\nabla\left(\operatorname{div}(\xi)-2 \lambda_{k}\left(r_{k}-v-\omega\right)\right)_{i, j}+a_{i, j} \xi_{i, j}=0
$$

where $a_{i, j}$ is the Lagrange multiplier. According to the complementary relaxation theorem, the $a_{i, j}$ satisfies

$$
a_{i, j}=\mid \nabla\left(\operatorname{div}(\xi)-2 \lambda_{k}\left(r_{k}-v-\omega\right)_{i, j} \mid\right.
$$

Using the semi implicit fixed point iterative algorithm to solve the variable $\xi$ in equation (13), the following can be got:

$\xi^{0}=0, \quad \xi_{\mathrm{i}, \mathrm{j}}^{\mathrm{n}+1}=\xi_{i, j}^{n}+\tau\left(\nabla\left(\operatorname{div}\left(\xi^{n}\right)-2 \lambda_{k}\left(r_{k}-v-\omega\right)\right)\right)_{i, j}-\mathfrak{\tau a}_{i, j}^{n} \xi_{i, j}^{n+1}$

In the above equation, $\xi_{i, j}^{n+1}$ is solved, and the final iteration format is obtained as follows:

$$
(\nabla \mathrm{u})_{i, j}=\left(\left(\partial_{x} u\right)_{i, j},\left(\partial_{y} u\right)_{i, j}\right)
$$

The discrete gradient operator is defined as:

$$
\left(\partial_{y} u\right)_{i, j}=\left\{\begin{array}{lr}
u_{i, j+1}-u_{i, j}, \text { if } j<N \\
0, & \text { if } j=N
\end{array}\right.
$$

The discrete divergence operator $\left(\operatorname{div}\left(\xi^{1}, \xi^{2}\right)\right)_{i, j}$ is defined as

$$
\left(\operatorname{div}\left(\xi^{1}, \xi^{2}\right)\right)_{i, j}=\left\{\begin{array}{ll}
\xi_{i, j}^{1}-\xi_{i, j-1}^{1}, & \text { if } 1<j<N \\
\xi_{i, j}^{1}, & \text { if } j=1 \\
-\xi_{i, j-1}^{1}, & \text { if } j=N
\end{array}+ \begin{cases}\xi_{i, j}^{2}-\xi_{i-1, j}^{2}, & \text { if } 1<i<N \\
\xi_{i, j}^{2}, & \text { if } i=1 \\
-\xi_{i-1, j}^{2}, & \text { if } i=N\end{cases}\right.
$$

Then the minimization problem (14) is solved. Since G norm is defined by the $L^{\infty}$ norm of function, the Euler-Lagrange equation of functional (14) cannot be solved [11], so the minimization problem (14) cannot be solved directly. In this paper, using the method of reference [12], the minimization problem (14) can be approximately as follows:

$$
\inf \left\{\lambda_{k}\left\|r_{k}-u-v-\omega\right\|_{L^{2}}^{2}+\left\|\nabla\left(\Delta^{-1} v\right)\right\|_{L^{2}}^{2}\right\}
$$

The Euler-Lagrange equation of functional (20) is as follows:

$$
\Delta^{-1} v=\lambda_{k}\left(u+v+\omega-r_{k}\right) \Rightarrow v=\Delta\left(\lambda_{k}\left(u+v+\omega-r_{k}\right)\right)
$$

The boundary condition is $\left.\frac{\partial\left(u+v+\omega-r_{k}\right)}{\partial n}\right|_{\partial \Omega}=0$, where $n$ represents the normal vector outside the boundary $\partial n$. Using the gradient descent method, the solution of equation (15) is equivalent to the steady-state solution of the following evolution equation

$$
\xi^{0}=0, \quad \xi_{i, j}^{\mathrm{n}+1}=\frac{\xi_{i, j}^{n}+\tau\left(\nabla\left(\operatorname{div}\left(\xi^{n}\right)-2 \lambda_{k}\left(r_{k}-v-\omega\right)\right)\right)_{i, j}}{1+\tau\left|\nabla\left(\operatorname{div}\left(\xi^{n}\right)-2 \lambda_{k}\left(r_{k}-v-\omega\right)\right)_{i, j}\right|}
$$

In the discrete case, the semi implicit finite difference scheme is used to solve (15), and the iterative scheme can be obtained as follows

$v^{0}=0, v_{i, j}^{n+1}=\frac{v_{i, j}^{n+1}+\tau\left(\lambda_{k}\left(\Delta\left(u+\omega+v^{n}-r_{k}\right)\right)_{i, j}\right)}{1+\tau}$

The discrete Laplacian operator is defined as $(\Delta \mathrm{u})_{i, j}=(\operatorname{div}(\nabla u))_{i, j}, \quad$ where $(\nabla .)_{i, j}$ and $(\operatorname{div})_{i, j}$ are calculated by equations (15) and (14), respectively [13].

Finally, the minimization problem (15) is solved. Using wavelet soft threshold (threshold is $4 \lambda_{\gamma_{2}}$ ) algorithm, the solution of the minimization problem (12) is approximately: 


$$
\omega+r_{k}-u-v-W S T\left(r_{k}-u-v, 4 \lambda_{k} r_{2}\right)
$$

where WST is the wavelet soft threshold operator, which is defined as $\operatorname{WST}(g, \delta)=\sum_{\left|g_{i, j}\right| \geq \delta} \hat{g_{i, j}} \varphi_{i, j}$, and $g=\sum \hat{g}_{i, j} \varphi_{i, j}$ is the wavelet decomposition of $g$.

In practical calculation, this paper first solves $\omega$, then $v$, and finally $u$ to speed up convergence. And the latest value is brought into the next iteration to speed up the convergence. The final algorithm is described as follows

Step1. Initialization. $u^{0}=v^{0}=\omega^{0}=0$.

Step2. Calculate $\omega^{n+1}$.

$\omega^{\mathrm{n}+1}=\mathrm{r}_{k}-u^{n}-v^{n}-W S T\left(\mathrm{r}_{k}-u^{n}-v^{n}, 4 \lambda_{K} \gamma_{2}\right)$

Step3. Calculate $v^{n+1}$.

$v_{i, j}^{n+1}=\frac{v_{i, j}^{n}+\tau\left(\lambda_{k}\left(\Delta\left(u^{n}+v^{n}+\omega^{n+1}-r_{k}\right)\right)_{i, j}\right)}{1+\tau}$

Step4. Calculate $\xi^{n+1}$. Initialize $\xi^{0}=0, n_{1}=0$.

The stop condition is $\left|\operatorname{div}\left(\xi_{i, j}^{n_{1}+1}\right)-\operatorname{div}\left(\xi_{i, j}^{n_{1}}\right)\right| \leq \varepsilon$.

Step5. Calculate $u^{n+1}$.

$u_{i, j}^{n+1}=r_{k, i, j}-v_{i, j}^{n+1}-\omega_{i, j}^{n+1}-\frac{1}{2 \lambda_{k}} \operatorname{div}\left(\xi_{i, j}^{n_{1}+1}\right)$.

The stop condition is $\max \left\{\left|u^{n+1}-u^{n}\right|,\left|v^{n+1}-v^{n}\right|\right\} \leq \varepsilon$.

The above algorithm describes how to solve coupling problems with fixed values, such as formula (10) - (12). In order to obtain the multi-scale variational decomposition, $k=-1$ is first set, and then the above algorithm is used to iterate over and over again, and $k \rightarrow k+1$ is reassigned in each step. The statement with the highest frequency in this algorithm is the calculation of $\nabla(\operatorname{dig}(\xi))$ and $\nabla(\operatorname{div}(\nabla u))$, which requires a two-layer loop, so the time complexity of this algorithm is $0(N)$.

$\left\{\begin{array}{l}\mathrm{v}_{t}=\Delta\left(\lambda_{k}\left(u+v+\omega-r_{k}\right)\right)-v \\ v(x, y, 0)=0,\left.\frac{\partial\left(u+v+\omega-r_{k}\right)}{\partial \vec{n}}\right|_{\partial \Omega}=0\end{array}\right.$

Figure 1 is the free-body diagram of VTOL aircraft on $O x y$ plane, which considers the vertical $\mathrm{y}$-axis and lateral $\mathrm{x}$-axis and ignores the exercise in front and back (ie, z-direction). $O x y$ is the inertial coordinate system.

\section{HYBRID BASE COMPRESSED SENSING ALGORITHM BASED ON VARIATIONAL DECOMPOSITION}

The structure component $u$ and the texture component $v$ obtained by the variational decomposition are reconstructed by the compressed sensing[14]. To further enhance the sparseness of $u$ and $v$, TFWBST and wave atom are used to represent the sparseness respectively.

TFWBST as a new multiscale geometric transform. deecompose the image by using $2 \mathrm{D}$ separable orthogonal wavelet bases and implement direction partition with dynamic window functions in the pseudo-polar grid. Therefore, a new multiscale geometric transform with tight framework is constructed.The new sparse bases are the optimally sparse for the image with various edges.

the reconstruction model is as follows

$$
\begin{gathered}
\hat{X}=\arg \min \left\|\Psi_{T}^{-1} u\right\|_{1}+\arg \min \left\|\Psi_{A}^{-1} v\right\|_{1} \\
\text { s.b. }\|y-\Phi(u+v)\|_{2} \leq \varepsilon
\end{gathered}
$$

$\hat{X}$ represents the reconstructed original image, $\Psi_{T}^{-1}$ and $\Psi_{A}^{-1}$ represent the inverse transform of TFWBST and wave atom respectively, and Gaussian matrix is used for the observation of $u$ and $v$ components.

Therefore, taking noise variance $\sigma^{2}$ as the stop criterion of iteration can effectively recover image structure information and remove the influence of noise.

Step1. Initialization: Initialize the reconstructed image $\hat{\boldsymbol{X}}=0$, where $k$ is the number of iterations, and $k=1$.

Step2. Random projection: the random projection sampling is carried out for the image $X=u+v$ after variational decomposition to obtain the observation value: $y=\Phi X$.

Step3. Carry out hard threshold iteration based on hybrid base;

$X_{k+1}=\Psi_{T}\left(\Gamma_{\mathrm{T}}\left(\Psi_{T}^{-1}\left(u_{k}+\Phi^{-1}\left(y-\Phi_{u_{k}}\right)\right)\right)\right)+\Psi_{A}\left(\Gamma_{\mathrm{T}}\left(\Psi_{A}^{-1}\left(v_{k}+\Phi^{-1}\left(y-\Phi v_{k}\right)\right)\right)\right)$ , where $\Phi^{-1}$ represents the inverse process of measurement matrix, and $\Gamma_{\mathrm{T}}(\boldsymbol{x})=\left\{\begin{array}{ll}\boldsymbol{x} & |\boldsymbol{x}| \geq \mathrm{T} \\ 0 & |\boldsymbol{x}|<\mathrm{T}\end{array}\right.$ stands for hard threshold operator.

Step4. $\hat{\boldsymbol{X}}=\hat{\boldsymbol{X}}+\boldsymbol{X}_{\boldsymbol{k}+1}$, Residual $v=y-\Phi \hat{X}$, number of iterations $\boldsymbol{k}=\boldsymbol{k}+1$.

Step5. Repeat steps 3-4 until the residual $\boldsymbol{\gamma}=\boldsymbol{\sigma}^{2}$ completes the iterative reconstruction. 


\section{EXPERIMENTAL RESULTS AND ANALYSIS}

\section{A Experiment setup}

Experimental test image: in this section, six test images fommonly used in the field of compressed sensing are selected or the experiment. The test image is shown in Fig.2, including Lena, Peppers, Leaves, Baboon, House and Barbara. The esolution of six images is $256 \times 256$. There are differences in the characteristics of these images, for example, Lena has both smooth areas and areas rich in detail and texture; Peppers are elatively smooth as a whole; Leaves have significant edges; Baboon has very rich details; House and Barbara have more texture areas. Therefore, the test results on these images can eflect the comprehensive performance of the algorithm.

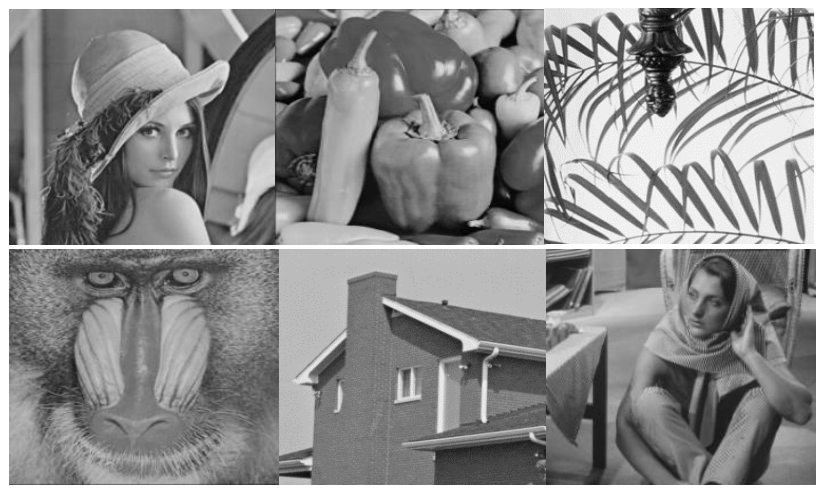

Fig. 2 The test images used in this section

Test images used in this chapter: Peppers, Leaves, Baboon, House, Barbara

Sampling rate setting: To comprehensively test the performance of the proposed algorithm under different sampling rates, experiments were carried out under the sampling rate of $20 \%, 30 \%$ and $40 \%$.

Experimental environment: The test experiment of this paper was completed on a desktop computer configured with Intel i7 CPU and 32 GB memory. The programming software used was Matlab 2014b.

\section{B Analysis of Experimental Results}

For images with rich texture details, such as Baboon, Leaves and Barbara images, the algorithm in this paper has no obvious advantages over WSGSR[15] algorithm in the reconstruction of image details, especially for the whisker area of Baboon image and the texture area of Barbara image. WSGSR algorithm has a little advantage in detail reconstruction compared with the algorithm in this paper, but there is noise interference in the reconstructed image. This is because the algorithm adopts the weighted sparse representation of non-local similar image block structure group, which can effectively reduce the loss of lowfrequency components of the image while recovering the highfrequency details of the image. However, since the reconstruction adopts the iterative hard threshold algorithm, the noise and artifact phenomena are more obvious. The algorithm in this paper is too smooth for the reconstruction of image details, resulting in unsatisfactory or missing reconstruction of some details.

In this paper, a multi-scale variational decomposition model with single parameter is proposed, and the relationship between this parameter and each component scale is discussed theoretically. This model has the following advantages for multi-scale image restoration and reconstruction. Multi-scale variational decomposition can decompose the image into a sequence of structures, textures and noises of different scales, which can be used for multi-scale image restoration and reconstruction conveniently. The decomposition is a ternary decomposition, which can separately extract the structure, texture and noise of the image at different scales.In this way, the texture of the reconstructed image can be better reconstructed, and the noise can be eliminated. Due to the multiscale and variational decomposition methods, the image feature information is fully taken into account, so there is almost no noise and artifact interference in the reconstruction.

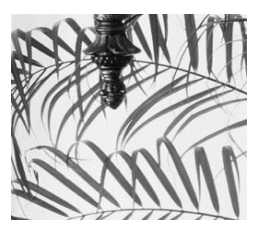

a)

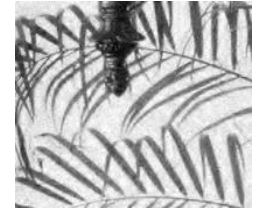

b)

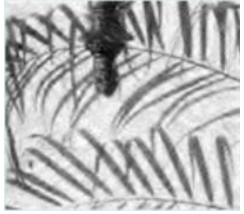

c)
Fig. 3 The CS reconstruction results of Leaves at sampling rate 0.2

a) Original image b) WSGSR algorithm reconstruction image c) Multiscale compression sensing of variational model algorithm reconstruction image

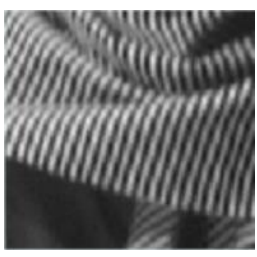

a)

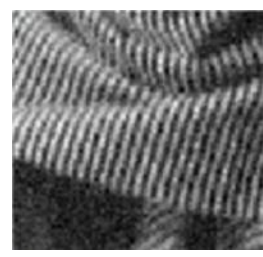

b)

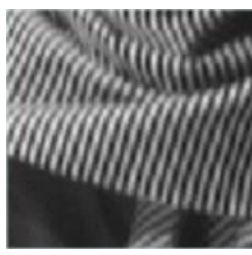

c)
Fig. 4 The CS reconstruction results of Leaves at sampling rate 0.2

a) Original image b) WSGSR algorithm reconstruction image c) Multiscale compression sensing of variational model algorithm reconstruction image

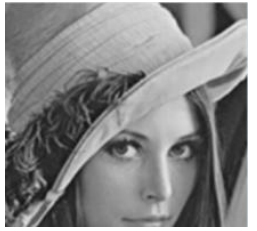

a)

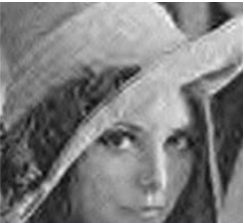

b)

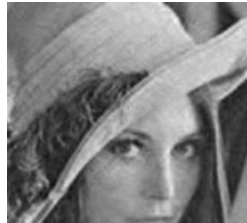

c)
Fig. 5 The CS reconstruction results of Leaves at sampling rate 0.2

a)Original image b) WSGSR algorithm reconstruction image

c) Multiscale compression sensing of variational model algorithm reconstruction image 


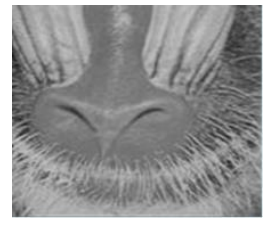

a)

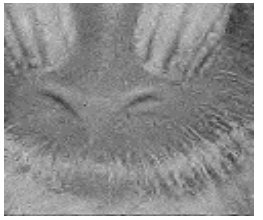

b)

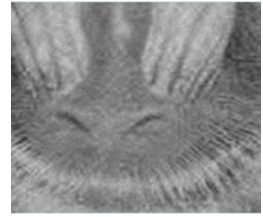

c)
Fig. 6 The CS reconstruction results of Leaves at sampling rate 0.2

a)Original image b) WSGSR algorithm reconstruction image c) Multiscale compression sensing of variational model algorithm reconstruction image

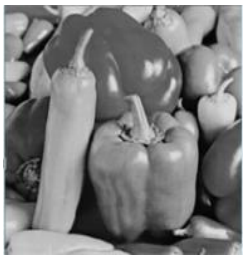

a)

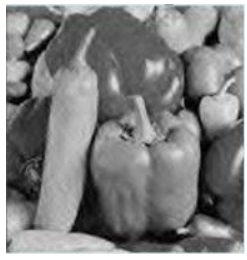

b)

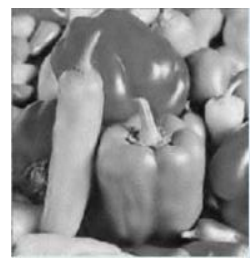

c)
Fig. 7 The CS reconstruction results of Leaves at sampling rate 0.2

a) Original image b) WSGSR algorithm reconstruction image c) Multiscale compression sensing of variational model algorithm reconstruction image

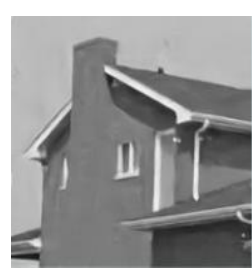

a)

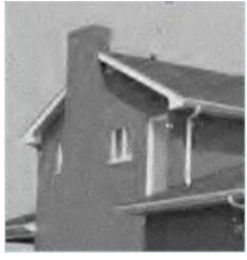

b)

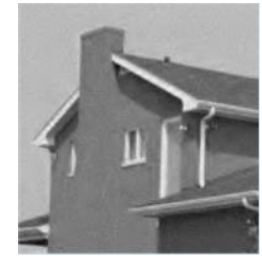

c)
Fig. 8 The CS reconstruction results of Leaves at sampling rate 0.2

a)Original image b) WSGSR algorithm reconstruction image c) Multiscale compression sensing of variational model algorithm reconstruction image

\section{CONCLUSION}

The variational image decomposition can decompose the image into different feature components by minimizing the energy functional, which can be used for image restoration and reconstruction. A multi-scale image compression sensing algorithm based on the variational framework is proposed. Firstly, a single parameter $(B V, G, E)$ ternary variational decomposition model is given, and the scale relationship between parameters and different feature components is theoretically analyzed. Then, the parameters of this model are selected as a binary sequence, and the variational decomposition of multi-scale $(B V, G, E)$ is obtained. The multi-scale variational decomposition can decompose the image into a sequence of image structure, texture and noise. The convergence of multi-scale decomposition is proved, and the numerical solution is given based on duality theory and alternating iterative algorithm. Finally, the variational decomposition of multi-scale $(B V, G, E)$ is applied to image restoration and reconstruction. The experimental results prove the correctness of the theoretical analysis, and show the effectiveness of this model in the multi-scale restoration and reconstruction of images, and the superiority of the model compared with other decomposition models.

When multi-scale decomposition is used for image restoration and reconstruction, noise is very easy to be reconstructed into the image along with texture at a certain scale, which reduces the quality of reconstructed image. This is mainly because there is no clean 'noise + texture' decomposition at a certain scale during image decomposition. Therefore, how to select appropriate space to model noise and texture is the focus of the subsequent variation-based multi-scale image restoration and reconstruction. Our idea is to integrate different statistics of texture and noise into the variational decomposition model, but we haven't implemented it yet, which will be the direction of our future research.

\section{REFERENCES}

[1] [1]Hu J , Baidoo E, Zhan L, et al. Computationally Efficient Compressed Sensing-Based Method via FG Nyström in Bistatic MIMO Radar with Array Gain-Phase Error Effect. International Journal of Antennas and Propagation, 2020, 2020(1):1-12.

[2] Cang S , Wang A . Research on Hyperspectral Image Reconstruction Based on GISMT Compressed Sensing and Interspectral Prediction. International Journal of Optics, 2020, 2020(12):1-11.

[3] Morimoto D , Hyodo T , Kamata K, et al. Navigator-triggered and breathhold 3D MRCP using compressed sensing: image quality and method selection factor assessment. 2020, In Press.

[4] Young C. Kim, Michael E. Fisher. Singular coexistence-curve diameters: Experiments and simulations. Chemical physics letters, 2005, 414(13):185-192.

[5] James D. E. Grant, Michael Kunzinger, Clemens Smann. Inextendibility of spacetimes and Lorentzian length spaces. Annals of Global Analysis and Geometry, 2019, 55(1):133-147.

[6] Kondori, Babak, Benzerga, A. Amine, Needleman, Alan. Discrete shear transformation zone plasticity. Extreme Mechanics Letters, 2016:S2352431616300062..

[7] Chen, Gang. Quantum spin ices and magnetic states from dipolaroctupolar doublets on the pyrochlore lattice. Aps March Meeting. American Physical Society, 2016.

[8] Candès, Emmanuel, Demanet, Laurent, Donoho, David. Fast Discrete Curvelet Transforms. Multiscale Modeling \& Simulation, 2006, 5(3).

[9] Liu Guojun, Feng Xiangchu, Zhang Xuande, et al. Threshold Algorithm of Texture Images with Gabors, 2009, 31(8):1791-1795.

[10] Easley G Labated R. Sparse directional image representations using the discrete Shearlet transform. Applied Computational Harmonic Analysis, 2008, 25 (1): 25-46.

[11] Rao J S . Euler-Lagrange Equations. Simulation Based Engineering in Solid Mechanics. Springer International Publishing, 2017.

[12] Li Jia, Gao Zhirong, Xiong Chengyi, et al. Image compressive sensing recovery based on weightedr structure group sparse representation. Journal of Communications, 2017, 038(002):196-202.

[13] Burt P J, Adelson E H . The Laplacian Pyramid as a Compact Image Code. Readings in Computer Vision, 1987, 31(4):671-679.

[14] ZhiQiang XU. Compressed sensing: a survey. Scientia Sinica, 2012, 42(9):865-877.

[15] Hongkun LI. Weak Fault Enhancement Method for Blade Crack by Using Stochastic Resonance. Journal of Mechanical Engineering, 2016, 52(1):94.

\section{Creative Commons Attribution License 4.0} (Attribution 4.0 International, CC BY 4.0)

This article is published under the terms of the Creative Commons Attribution License 4.0

https://creativecommons.org/licenses/by/4.0/deed.en_US 